\title{
Fatores Associados ao Parto Vaginal em Gestantes de Alto Risco Submetidas à Indução do Parto com Misoprostol
}

\author{
Factors Associated with Vaginal Delivery in High-Risk Pregnant Women \\ Submitted to Labor Induction with Misoprostol \\ Zuleika Studart Sampaio ${ }^{1}$, Carlos Augusto Alencar Júnior ${ }^{1}$, \\ Francisco Edson de Lucena Feitosa ${ }^{1}$, Melania Maria Ramos de Amorim²
}

\begin{abstract}
RESUMO
Objetivos: determinar os principais fatores associados ao parto transpelvino em gestantes de alto-risco submetidas à indução do parto com misoprostol vaginal (50 $\mu \mathrm{g})$.

Métodos: trata-se da análise secundária de um ensaio clínico aberto, não randomizado, incluindo 61 gestantes de alto-risco internadas na Enfermaria de Patologia Obstétrica da Maternidade Escola Assis Chateaubriand, em Fortaleza (CE). Todas tinham idade gestacional de mais de 37 semanas, feto único com boa vitalidade e escores de Bishop menores ou iguais a 7. Utilizou-se a dose de $50 \mu \mathrm{g}$ de misoprostol via vaginal, repetindo-se a cada seis horas, até o máximo de quatro doses. Realizou-se análise uni e multivariada para determinação da associação entre parto vaginal (variável dependente) e variáveis independentes (preditoras), construindo-se curvas ROC para paridade e escores de Bishop.

Resultados: encontrou-se associação significativa do parto vaginal com a paridade (um ou mais partos anteriores), escore de Bishop $\geq 4$ e intervalo entre indução e parto menor que 6 horas, ao passo que a taquissistolia reduziu a chance de parto vaginal. Realizou-se análise de regressão logística múltipla para avaliar cada um desses fatores independentemente, persistindo fortemente associados ao parto vaginal o escore de Bishop $\geq 4(O R=3,3 ;$ IC 95\% $=2,15-4,45)$ e a paridade $\geq 1(O R=5,41 ;$ IC 95\% = 4,18-6,64). Pela curva ROC para os escores de Bishop e a paridade como determinantes do parto vaginal, encontrou-se um valor preditivo positivo de $100 \%$, com sensibilidade de $63,2 \%$. A área sob a curva foi de $86,8 \%$, significativamente superior a $50 \%(p=0,023)$.

Conclusões: os fatores preditivos mais importantes para parto vaginal após indução com misoprostol foram a paridade (um ou mais partos) e escores de Bishop $\geq 4$. Estes fatores devem ser levados em consideração na escolha de diferentes esquemas e doses de misoprostol, tanto para preparo cervical como indução do parto.
\end{abstract}

PALAVRAS-CHAVE: Indução do parto. Prostaglandinas. Trabalho de parto.

\section{Introdução}

O misoprostol, análogo sintético da $\mathrm{PGE}_{1}$, vem sendo usado para indução do parto desde

${ }^{1}$ Maternidade-Escola Assis Chateaubriand - Universidade Federal do Ceará

${ }^{2}$ Instituto Materno-Infantil de Pernambuco (IMIP) - Recife Correspondência:

Melania Maria Ramos de Amorim

Rua Neuza Borborema de Sousa, 300 - Bairro Santo Antônio

58103-313 - Campina Grande - PB

e-mail: melamorim@uol.com.br
$1991^{1}$. Diversos ensaios clínicos têm comprovado sua efetividade em comparação a outros métodos de indução, como ocitocina, prostaglandina $\mathrm{E}_{2}$ vaginal, sonda de Foley e solução salina extraamniótica $^{2,3}$. Na revisão sistemática disponivel na Biblioteca Cochrane, conclui-se que o misoprostol é mais efetivo que os métodos convencionais para amadurecimento do colo e indução do parto ${ }^{3}$. Diversos esquemas posológicos e vias de administração estão disponíveis, mas a via vaginal oferece vantagens em relação à oral, devido a perfil farmacocinético mais favorável ${ }^{4,5}$. A dose de 
$50 \mu \mathrm{g}$ via vaginal resulta em intervalo mais curto até o parto, embora tenha sido relatado aumento da incidência de taquissistolia e hiperatividade uterina $^{3,6}$. Intervalos entre três e seis horas têm sido utilizados, porém doses e intervalos geralmente se baseiam nos resultados dos estudos farmacocinéticos, bem como em estudos clínicos comparando a eficácia dos diversos esquemas ${ }^{7}$. Entretanto, poucos estudos têm sido publicados considerando possiveis fatores, maternos ou gestacionais, que podem interferir nas características dose-resposta do misoprostol utilizado por via vaginal $^{8}$.

Nesse sentido, Wing et al. ${ }^{8}$, publicaram em 2002 estudo analisando os diversos fatores afetando a probabilidade de sucesso da indução depois da aplicação do misoprostol intravaginal para dilatação cervical e indução do parto. Os autores analisaram diversas variáveis, como indicação da indução, idade, idade gestacional, paridade, peso, altura, índice de massa corpórea, dilatação cervical e escore de Bishop iniciais, dose total de misoprostol, necessidade de uso de ocitocina, anestesia peridural e peso ao nascer. Nesse estudo, definiu-se sucesso da indução como a ocorrência de parto vaginal dentro de 24 horas depois da dose inicial de misoprostol. Encontraram associação estatisticamente significante da idade gestacional, paridade, dilatação cervical e escore de Bishop como preditores do sucesso da indução, porém depois do ajuste para possíveis fatores de confusão em modelo de regressão logística múltipla, persistiram como fatores associados ao sucesso da indução apenas as características clínicas paridade, dilatação cervical inicial e idade gestacional na admissão.

Em revisão nos bancos de dados MEDLINE, LILACS e sciELO, utilizando os termos "Misoprostol" e "Labor", encontramos escassas referências avaliando os fatores associados ao sucesso da indução com misoprostol ${ }^{9,10}$. Alguns estudos incluem diversos outros métodos de indução ${ }^{11}$, ao passo que a maior parte dos estudos mais recentes tem avaliado basicamente o papel da ultrasonografia transvaginal para predição de indução bem-sucedida ${ }^{12,13}$

Entretanto, o misoprostol vem sendo amplamente utilizado em nosso meio, e diversos ensaios clínicos estão sendo ou foram conduzidos em nosso serviço ${ }^{14-16}$. Embora a droga seja comprovadamente eficaz para indução do parto, é importante identificar os fatores que podem afetar sua eficácia, para propor esquemas alternativos para aumentar a probabilidade de parto vaginal, estratégia que seria particularmente importante no
Brasil, onde a incidência de operação cesariana vem alcançando patamares inaceitáveis ${ }^{17}$.

Assim, o presente estudo foi desenhado com o objetivo de determinar os principais fatores associados ao parto transpelvino em gestantes de alto risco submetidas à indução do parto com misoprostol vaginal (50 $\mu \mathrm{g}$ ) na Maternidade-Escola Assis Chateaubriand, em Fortaleza (Ceará).

\section{Pacientes e Métodos}

O presente estudo representa uma análise secundária dos dados de um ensaio clínico aberto e não randomizado realizado na Enfermaria de Patologia Obstétrica da Maternidade-Escola Assis Chateaubriand da Universidade Federal do Ceará (MEAC-UFC), no período de março de 2002 a março de $2003^{14}$. Este estudo respeitou as normas da Declaração de Helsinque e da resolução n ${ }^{\circ}$ 196/ 96 do Conselho Nacional de Saúde para pesquisa em seres humanos, tendo sido aprovado pelo Comitê de Ética em Pesquisa da Universidade Federal do Ceará. Todas as pacientes foram devidamente informadas sobre os objetivos da pesquisa e concordaram em participar do estudo, assinando o Termo de Consentimento Livre e Esclarecido.

O estudo incluiu 61 gestantes de alto risco, com gravidez única, idade gestacional maior ou igual a 37 semanas, feto vivo, em apresentação cefálica, com peso fetal estimado por ultra-sonografia $<4000 \mathrm{~g}$, índice de líquido amniótico maior que $5 \mathrm{~cm}$, cardiotocografia basal anteparto normal e escore de Bishop menor ou igual a sete. Foram excluídas as gestantes com cesárea prévia, cicatriz uterina por miomectomia, apresentação anômala, provas de vitalidade fetal alteradas (cardiotocografia, perfil biofísico fetal e Doppler), restrição de crescimento fetal, gestação múltipla, epidemiologia para asma, reações adversas ao uso de prostaglandinas ou alguma contra-indicação para o parto vaginal.

Realizou-se, obrigatoriamente, exame de cardiotocografia basal anteparto imediatamente antes do uso do misoprostol. A administração do medicamento foi realizada na Enfermaria de Patologia Obstétrica, introduzindo-se $50 \mu \mathrm{g}$ de misoprostol no fundo de saco vaginal posterior, durante o exame de toque vaginal bidigital, pelo médico-residente. Cada dose de $50 \mu \mathrm{g}$ foi obtida pelo fracionamento de um comprimido de $200 \mu \mathrm{g}$ de Cytotec $^{\circledR}$ - Searle em quatro partes iguais. A seguir, as pacientes foram acompanhadas, monitorizando-se a presença de contrações 
uterinas a cada hora, até o parto. O intervalo entre as doses foi de seis horas, até o início do trabalho de parto, obtendo-se padrão contrátil satisfatório, ou o máximo de quatro doses. Considerou-se padrão contrátil satisfatório a presença de pelo menos três contrações de 40 ou mais segundos de duração, em 10 minutos de observação. Após seis horas da administração da última dose do medicamento e na ausência de trabalho de parto, o tratamento era suspenso e o caso deveria ser reavaliado.

Após desencadeamento do trabalho de parto, as gestantes passaram a ser acompanhadas pelos médicos de plantão do Centro Obstétrico da MEAC-UFC, monitorizando-se a dinâmica uterina e a freqüência cardíaca fetal. A ausculta fetal foi realizada de forma intermitente em todos os casos, a cada 15 minutos, antes, durante e depois das contrações, quando presentes. Cardiotocografia foi realizada de forma intermitente a cada duas horas, até o parto, ou ainda se considerado necessário pelo médico-assistente para documentação de distúrbios da contratilidade uterina e da freqüência cardíaca fetal. A dinâmica era verificada clinicamente, a cada 30 minutos.

A variável dependente analisada foi o tipo de parto (vaginal ou cesárea), determinando-se a associação entre o parto vaginal e as variáveis independentes: indicação da indução do parto, idade, paridade, idade gestacional, peso fetal estimado pela ultra-sonografia, índice do líquido amniótico, escore de Bishop inicial, tempo transcorrido entre início da indução e parto, número de doses de misoprostol, presença de taquissistolia, líquido amniótico meconial e peso ao nascer.

Em relação à indicação de indução do parto, anotou-se a condição clínica e/ou obstétrica preponderante impondo a resolução da gestação, não impeditiva do parto vaginal. Definiu-se gestação prolongada na presença de idade gestacional maior ou igual a 41 semanas. Pré-eclâmpsia foi definida de acordo com os critérios do National High Blood Pressure Working Education Report (2000) ${ }^{18}$. Cada uma destas condições representou uma variável categórica dicotômica, tipo sim ou não, para finalidade de análise estatística.

Registrou-se a idade materna em anos completos, determinando-se o ponto de corte como menor que 20 e maior ou igual a 20 anos. Anotou-se também a paridade, considerando-se nulíparas e pacientes com um ou mais partos anteriores. A idade gestacional foi estimada pela data da última menstruação, quando conhecida e confiável, e/ou pela ultra-sonografia realizada na primeira metade da gestação ou na segunda metade da gestação, desde que compativel com a data da última menstruação e confirmada pela avaliação do recém-nascido pelo método de Capurro. Para análise estatística, a idade gestacional foi codificada como menor ou igual a 40 e maior que 40 semanas.

$O$ peso fetal estimado pela ultra-sonografia foi calculado automaticamente pelo aparelho, considerando os parâmetros de biometria fetal, por meio da seguinte equação de regressão: peso = 1,3596-0,00386 x (circunferência abdominal (CA)) $\mathrm{x}$ (comprimento do fêmur $(\mathrm{CF})+0,0064 \mathrm{x}$ (circunferência cefálica $(\mathrm{CC})+0,00061 \times$ (diâmetro biparietal (DBP) $\mathrm{x}(\mathrm{CA})+0,0424 \mathrm{x}(\mathrm{CA})+0,174 \mathrm{x}$ $(\mathrm{CF})^{19}$. Esta variável foi anotada em gramas e incluída no modelo de regressão logística como variável contínua.

$\mathrm{O}$ índice do líquido amniótico foi determinado dividindo-se o útero em quatro quadrantes, por duas linhas perpendiculares que se cruzam ao nível da cicatriz umbilical, e somando-se as dimensões verticais dos maiores bolsões ${ }^{20}$, sendo expresso em centímetros e incluído no modelo de regressão como variável contínua.

Utilizou-se o escore de Bishop modificado (NHS, 1996) ${ }^{21}$, com os parâmetros relacionados avaliados antes do início da indução pelo toque vaginal bidigital, observando-se dilatação, posição, consistência, tamanho e altura da apresentação. Para análise estatística, consideraram-se duas categorias: Bishop maior ou igual a quatro, e menor que quatro.

O tempo transcorrido entre o início da indução e o parto foi calculado a partir da administração do primeiro comprimido até o nascimento, sendo expresso em horas. Consideraramse duas categorias: menor que seis horas e maior ou igual a seis horas. Registrou-se ainda o número total de doses de misoprostol utilizadas até a deflagração do trabalho de parto. Esta variável foi codificada como uma dose e maior ou igual a duas doses.

Definiu-se taquissistolia como a presença de pelo menos cinco contrações uterinas por dois períodos consecutivos de dez minutos ${ }^{22}$, categorizando-se a variável dicotômica como sim ou não. Também foi anotada a presença ou não de mecônio no líquido amniótico. O peso ao nascer foi expresso em gramas, e analisado como variável contínua no modelo de regressão logística múltipla.

A análise estatística foi realizada no programa SPSS versão 10.0 para Windows. Realizou-se inicialmente análise univariada para determinar a associação entre cada uma das variáveis independentes e a variável dependente. Para testar a 
associação entre as indicações de indução e o parto vaginal, utilizou-se o teste $\chi^{2}$ de Pearson, com nível de significância de 5\%. Para análise da associação entre as outras variáveis independentes e o parto vaginal, utilizou-se a razão de risco (RR) como medida do risco relativo, calculando-se o intervalo de confiança a 95\% (IC 95\%). Às categorias de referência, atribuiu-se o risco padrão de 1,00.

Em seguida, realizou-se análise multivariada para determinação do risco ajustado de parto vaginal. As variáveis dependentes foram recodificadas binariamente $(0=\operatorname{sim} / 1=$ não $)$, sendo que as variáveis peso fetal estimado, índice do líquido amniótico e peso ao nascer entraram no modelo como variáveis contínuas. Procedeu-se então à análise multivariada por meio de um modelo selecionado por análise passo a passo, utilizando-se o comando backward do programa estatístico. Definiu-se o modelo final com base nas variáveis independentes que persistiram associadas ao desfecho, com erro alfa menor que 5\%, e no melhor valor preditivo para parto vaginal. Determinou-se o odds ratio (OR) como estimativa do risco ajustado, bem como o IC 95\% para cada uma destas variáveis.

Definidas as variáveis mais fortemente associadas ao parto vaginal, foram calculados os parâmetros de acurácia (sensibilidade, especificidade, valor preditivo positivo, valor preditivo negativo, razão de verossimilhança positiva e razão de verossimilhança negativa) para as variáveis pré-indução (paridade e escore de Bishop), construindo-se ainda curvas ROC (receiver operating characteristic) para o escore de Bishop, tanto isolado como associado à paridade.

\section{Resultados}

As características da amostra estudada já foram descritas em estudo anterior ${ }^{14}$. Resumidamente, a idade variou entre 12 e 41 anos (média de 24,3 anos), a paridade entre zero e oito (mediana de zero), com percentual de nulíparas de $62,3 \%$. A média de idade gestacional foi de 39,9 semanas e a mediana dos escores de Bishop foi de três, encontrando-se 55,7\% dos casos com escores de Bishop abaixo de quatro.

A freqüência de parto vaginal observada no presente estudo foi de 54, 1\% (33 casos). As principais indicações de indução foram gestação prolongada (34 casos) e pré-eclâmpsia (17 casos). A freqüência de parto vaginal foi de 55,9\% nos casos de gestação prolongada, 58,8\% nos casos de préeclâmpsia e 50\% nos casos restantes $(p=0,91)$.
$\mathrm{Na}$ análise univariada, encontraram-se como fatores significativamente associados ao parto vaginal a paridade maior ou igual a um $(\mathrm{RR}=$ 2,09; IC 95\% = 1,35-3,24), o escore de Bishop inicial maior ou igual a quatro $(\mathrm{RR}=1,80$; IC $95 \%=$ $1,14-2,85)$ e o tempo transcorrido entre indução e parto menor que seis horas $(\mathrm{RR}=1,89$; IC 95\% = 1,34-2,67). A presença de taquissistolia associouse a redução significativa da freqüência de parto vaginal $(R R=0,21 ;$ IC 95\% = 0,06-0,77). Não houve associação do parto vaginal com as demais variáveis (idade, idade gestacional, número de doses de misoprostol e presença de mecônio) (Tabela 1).

$\mathrm{Na}$ análise de regressão logística múltipla, persistiram com associação significativa ao parto vaginal apenas o escore de Bishop maior ou igual a quatro $(\mathrm{OR}=3,3$; IC $95 \%=2,15-4,45)$ e a paridade maior que um (OR = 5,41; IC 95\% = 4, 18-6,64). Verificou-se redução significativa da chance de parto vaginal na presença de taquissistolia, mas selecionou-se um modelo final incluindo somente o escore de Bishop e a paridade, tanto por representarem variáveis pré-indução, com maior utilidade clínica, como porque compuseram um modelo que predisse corretamente o parto vaginal em $78,8 \%$ dos casos, não havendo portanto vantagem em se incluir a taquissistolia.

Analisando-se os parâmetros de acurácia para o escore de Bishop maior ou igual a quatro e a paridade maior que um, observaram-se sensibilidade, especificidade, valores preditivo positivo e negativo, razão de verossimilhança positiva e razão de verossimilhança negativa semelhantes, com acurácia global de respectivamente 65,6 e 68,9\% (Tabela 2).

Construíram-se curvas ROC para as variáveis preditoras do parto vaginal (escore de Bishop e paridade). Quando se analisou a curva para o escore de Bishop isolado, observou-se área sob a curva de $71 \%$, estatisticamente superior a $50 \%$ $(\mathrm{p}=0,005)$, tendo portanto poder discriminatório para o parto vaginal. Evidenciou-se, também, que o melhor ponto de corte do escore de Bishop como determinante do parto vaginal é quatro, apresentando o melhor contrabalanço entre sensibilidade e especificidade e com maior proximidade do canto superior esquerdo do gráfico (Figura 1).

Por outro lado, analisando-se conjuntamente os dois fatores por meio de curva ROC, observou-se área sob a curva de $86 \%$, também significativamente superior a $50 \%(\mathrm{p}=0,023)$, com maior sensibilidade $(63,2 \%)$ e valor preditivo positivo de $100 \%$, corroborando assim o resultado do modelo de regressão logística múltipla. 
Tabela 1 - Fatores associados ao parto vaginal após indução com misoprostol. Análise univariada.

\begin{tabular}{|c|c|c|c|c|c|c|}
\hline \multirow[t]{2}{*}{ Variável } & \multicolumn{2}{|c|}{ Parto vaginal } & \multicolumn{2}{|c|}{ Cesárea } & \multirow[t]{2}{*}{$\mathrm{RR}$} & \multirow[t]{2}{*}{ IC 95\% } \\
\hline & $\mathrm{n}$ & $\%$ & $\mathrm{n}$ & $\%$ & & \\
\hline \multicolumn{7}{|l|}{ Idade } \\
\hline$\geq 20$ anos & 27 & 58,7 & 19 & 41,3 & 1,26 & $0,7-2,28$ \\
\hline$<20$ anos & 07 & 46,7 & 08 & 53,3 & 2,09 & \\
\hline \multicolumn{7}{|l|}{ Paridade } \\
\hline Um ou mais partos & 19 & 82,6 & 04 & 17,4 & 1,00 & $1,35-3,24$ \\
\hline Nulíparas & 15 & 39,5 & 23 & 60,5 & 1,34 & \\
\hline \multicolumn{7}{|l|}{ Idade gestacional } \\
\hline$\leq 40$ semanas & 31 & 57,4 & 23 & 42,6 & 1,00 & $0,55-3,25$ \\
\hline$>40$ semanas & 03 & 42,9 & 04 & 57,1 & 1,80 & \\
\hline \multicolumn{7}{|l|}{ Bishop inicial } \\
\hline$\geq 4$ & 20 & 74,1 & 07 & 25,9 & 1,00 & $1,14-2,85$ \\
\hline$<4$ & 14 & 41,2 & 20 & 58,8 & 1,89 & \\
\hline \multicolumn{7}{|c|}{ Tempo entre indução e o parto } \\
\hline$<6$ horas & 10 & 90,9 & 01 & 9,1 & 1,00 & $1,34-2,67$ \\
\hline$\geq 6$ horas & 24 & 48,0 & 26 & 52,0 & 1,57 & \\
\hline \multicolumn{7}{|l|}{ Doses de misoprostol } \\
\hline Uma & 29 & 60,4 & 19 & 39,6 & 1,00 & $0,76-3,24$ \\
\hline Duas ou mais & 05 & 31,5 & 08 & 61,5 & & \\
\hline \multicolumn{7}{|l|}{ Taquissistolia } \\
\hline Sim & 02 & 14,3 & 12 & 85,7 & 0,21 & $0,06-0,77$ \\
\hline Não & 32 & 68,1 & 15 & 31,9 & & \\
\hline \multicolumn{7}{|l|}{ Presença de mecônio } \\
\hline Sim & 01 & 20,0 & 04 & 80,0 & 0,34 & $0,06-1,99$ \\
\hline Não & 33 & 58,9 & 23 & 41,1 & & \\
\hline
\end{tabular}

$\mathrm{RR}=$ razão de risco.

IC $95 \%$ = intervalo de confiança a $95 \%$.

Tabela 2 - Acurácia do escore de Bishop e da paridade para predição do parto vaginal.

Parâmetro de acurácia

Escore de Bishop

$\geq 4$

\section{Sensibilidade}

Especificidade

Valor Preditivo Positivo

Valor Preditivo Negativo

Razão de Verossimilhança Positiva

Razão de Verossimilhança Negativa

Acurácia

$\begin{array}{cc}58,8 \% & 55,9 \% \\ 74,1 \% & 85,2 \% \\ 74,1 \% & 82,6 \% \\ 58,8 \% & 60,5 \% \\ 2,48 & 3,29 \\ 0,48 & 0,51 \\ 65,6 \% & 68,9 \%\end{array}$




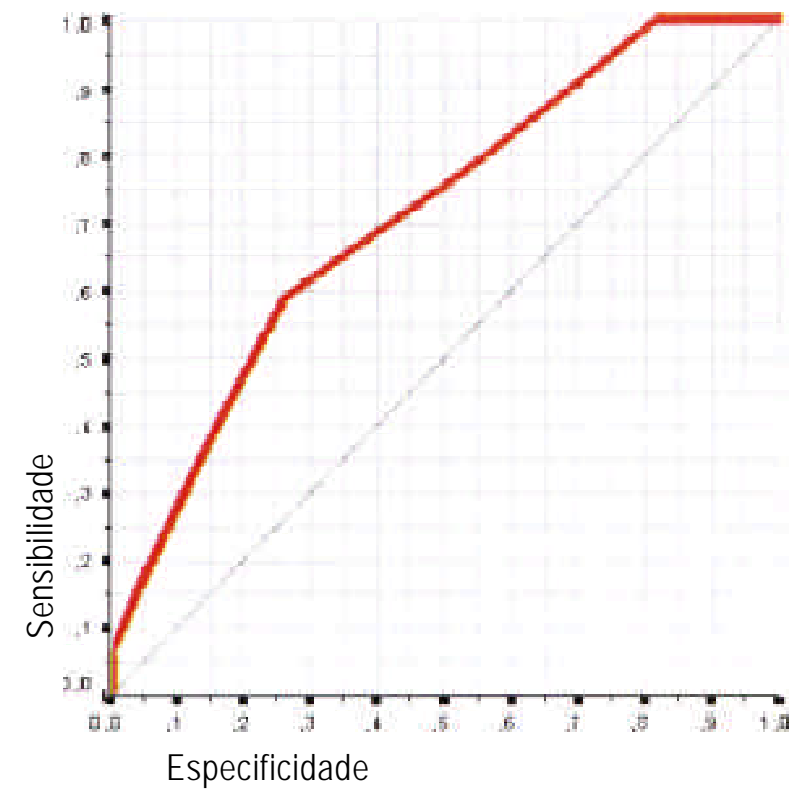

Figura 1 - Curva ROC para o escore de Bishop.
Área sob a curva ROC

0,71

$$
\begin{gathered}
\text { Erro-padrão } \\
0,066
\end{gathered}
$$

$\stackrel{p}{0,005}$

IC $95 \%$

\section{Discussão}

No presente estudo, os fatores mais fortemente associados com a ocorrência de parto vaginal após indução com misoprostol foram paridade (um ou mais partos anteriores) e escore de Bishop maior ou igual a quatro. Estes fatores persistiram significativamente associados ao parto transpelvino quando se realizou análise de regressão logística múltipla, com controle de outras variáveis, como idade, idade gestacional, estimativa do peso fetal, índice do líquido amniótico, tempo transcorrido entre início da indução e parto, número de doses de misoprostol, presença de taquissistolia, líquido amniótico meconial e peso ao nascer. O modelo associando escore de Bishop igual ou superior a 4 e paridade maior ou igual a 1 predisse corretamente $78,8 \%$ dos partos vaginais, com área sob a curva ROC de 86\%.

Esses achados não são surpreendentes, uma vez que tanto o escore de Bishop como a paridade têm sido apontados como preditores do parto vaginal em diversos outros estudos que analisam os fatores associados à indução bem sucedida do trabalho de parto ${ }^{9-13,23-25}$.

No próprio estudo original de Bishop ${ }^{26}$, descreveram-se que multíparas com escore cervical maior que 8 tinham a mesma probabilidade de parto vaginal que pacientes em trabalho de parto espontâneo. Desde então, vários artigos têm sido publicados analisando os fatores preditivos para sucesso na indução do parto. Entre os mais recentes, destaca-se o estudo de Watson et al. ${ }^{11}$, comparando critérios clínicos de avaliação cervical (posição, consistência, dilatação, apagamento e altura da apresentação), paridade e a medida ecográfica do colo uterino. Os autores realizaram análise de regressão logística para determinar os fatores que melhor predissessem o comprimento da fase latente do parto. Houve correlação significativa entre a avaliação clínica do colo e a avaliação ultra-sonográfica, porém apenas a dilatação cervical predisse independentemente o comprimento da fase latente do parto, sem efeito adicional significativo da avaliação ecográfica e da paridade.

Na verdade, a maioria dos estudos publicados nos últimos anos tem considerado o papel da ultra-sonografia transperineal ou transvaginal para avaliação cervical e predição do sucesso da indução do parto ${ }^{9,12,13}$. Teoricamente, a medida ecográfica do colo representa uma forma mais acurada de avaliação da cérvice uterina em relação ao exame digital, porque a porção supravaginal do colo é difícil de avaliar digitalmente, além do que o apagamento é uma informação subjetiva e pode variar consideravelmente entre os examinadores, sobretudo quando o colo está fechado. A medida do colo pela ultra-sonografia, além de representar adequadamente o processo de apagamento, constitui avaliação quantitativa e facilmente reproduzivel ${ }^{27}$.

Não obstante essas considerações, os estudos clínicos são discrepantes quando comparam a medida ecográfica do colo com o escore de Bishop ou a dilatação cervical. Gonem et al. ${ }^{12}$ analisaram 86 pacientes induzidas com ocitocina ou prostaglandina $\mathrm{E}_{2}$, comparando o escore de Bishop com o comprimento cervical pela ultra-sonografia transvaginal. Em um modelo de regressão logística, apenas paridade e escore de Bishop apresentaram correlação significante com indução bem sucedida e com a duração do parto.

Boozarjomehri et al. ${ }^{24}$ encontraram que a presença do funil cervical na ultra-sonografia representava melhor fator preditivo de duração da fase latente, mas não da duração total do trabalho de parto. Entretanto, Paterson-Brown et al. ${ }^{23}$ não encontraram correlação entre o comprimento cervical e a duração do trabalho de parto, nem tampouco entre comprimento cervical e parto vaginal. Em outra direção, Rane et al. ${ }^{13}$ avaliaram 382 gestações prolongadas, das quais 311 tiveram 
parto normal. Em nulíparas a incidência de cesárea foi de $28,1 \%$, versus $8,9 \%$ em multíparas. A análise de regressão logística demonstrou que o comprimento cervical e a paridade eram fatores preditivos independentes para cesariana, sendo que a chance de cesariana aumentava em torno de $10 \%$ com cada aumento de $1 \mathrm{~mm}$ no comprimento cervical, acima do comprimento médio de $20 \mathrm{~mm}$ para nulíparas e $18 \mathrm{~mm}$ para multíparas. A análise das curvas ROC evidenciou ainda que o comprimento cervical era melhor que o escore de Bishop para predição de cesariana (áreas sob a curva de respectivamente 0,72 versus 0,68 ).

Por outro lado, a maior parte dos estudos acima descritos incluíram apenas gestações em que o trabalho de parto foi induzido com ocitocina e/ou prostaglandina $\mathrm{E}_{2}$. Na literatura consultada, destacam-se poucos artigos avaliando as variáveis associadas ao sucesso na indução do parto com misoprostol, e a maioria destes diz respeito ao uso da ultra-sonografia transvaginal para determinação do comprimento cervical ${ }^{9,10,12,13,23-25}$.

O primeiro estudo publicado avaliando a medida ecográfica do comprimento do colo como fator preditivo tanto da duração do parto como da probabilidade de parto vaginal foi o de Ware e Raynor ${ }^{9}$, analisando 77 mulheres, das quais $58 \%$ receberam ocitocina e $42 \%$ misoprostol vaginal, na dose de 50 $\mu \mathrm{g}$ a cada quatro horas. A freqüência de parto vaginal foi de $69 \%$. Houve correlação linear do escore de Bishop e do comprimento cervical mensurado pela ultra-sonografia com a duração do parto. Tanto o escore de Bishop como a medida do comprimento cervical pela ultra-sonografia predisseram corretamente a duração do parto e a probabilidade de parto vaginal, porém após regressão logística múltipla apenas o comprimento cervical e a paridade persistiram como preditores independentes do tipo de parto. Esse modelo predisse corretamente o parto vaginal em $88 \%$ dos casos.

Em outro estudo, colombiano, publicado em 2000 , também se analisou o papel da cervicometria na predição de êxito pós-indução do parto com misoprostol, porém o desfecho analisado não foi o parto vaginal, e sim o intervalo entre início da indução e parto $^{10}$. Em 118 pacientes que receberam misoprostol vaginal na dose de $50 \mu \mathrm{g}$ a cada seis horas, obteve-se freqüência de parto vaginal de $82,2 \%$. Realizou-se análise de regressão logística múltipla, incluindo variáveis como paridade, cervicometria, idade da paciente e idade gestacional. A única variável preditora do intervalo entre início da indução e parto vaginal foi a cervicometria, considerando-se o ponto de corte de $4 \mathrm{~cm}(\mathrm{p}=0,03)$.
Análise mais sofisticada, considerando variáveis clínicas, foi realizada por Wing et al. ${ }^{8}$, incluindo 1373 gestações em que se utilizou o misoprostol vaginal para preparo cervical e indução do parto. Conforme já discutimos anteriormente, os autores encontraram associação significativa entre parto vaginal e as seguintes variáveis: paridade $(\mathrm{OR}=2,4 ; \mathrm{IC} 95 \%=2,0-3,0)$, dilatação cervical inicial $(\mathrm{OR}=1,7$; IC 95\% $=1,4-2,1)$ e idade gestacional estimada $(\mathrm{OR}=1,3$; IC 95\% $=1,1$ 1,6). Na análise de regressão logística, embora a dilatação cervical tenha persistido associada ao parto vaginal, o escore de Bishop não se manteve como fator preditivo importante, muito possivelmente porque tanto o escore de Bishop como a dilatação estão fortemente associados um ao outro, podendo ter ocorrido colinearidade.

Em nosso estudo, apesar de termos incluído apenas 61 pacientes, todas receberam misoprostol e não foi utilizada outra droga para preparo cervical ou indução do parto. Além disso, a freqüência de operação cesariana foi relativamente elevada, o que pode ser atribuído pelo menos parcialmente à maioria de pacientes nulíparas e com escores de Bishop abaixo de quatro na população estudada. Em outro estudo com a mesma casuística, tivemos oportunidade de discutir outros fatores possivelmente associados com essa taxa elevada de cesarianas ${ }^{14}$, porém o fato é que essas características da amostra permitiram um poder suficiente para evidenciar diferenças que, eventualmente, podem não ter sido demonstradas em estudos com taxas mais baixas de operação cesariana.

Até onde chega o nosso conhecimento, este é o primeiro estudo brasileiro avaliando variáveis clínicas associadas ao parto vaginal após indução com misoprostol. Embora outros ensaios clínicos em grandes maternidades brasileiras tenham sido publicados utilizando misoprostol para indução do parto com feto vivo ${ }^{28-30}$, não se consideraram os fatores preditivos para indução bem sucedida.

Julgamos importante a validação do escore de Bishop na predição do parto vaginal, principalmente por se tratar de método clínico e de fácil utilização em todas as maternidades. Destacamos, outrossim, que utilizamos um escore modificado, em que se substitui a avaliação do apagamento cervical, excessivamente subjetiva, pelo comprimento do colo mensurado clinicamente. Essa avaliação quantitativa não tem sido adequadamente avaliada em outros estudos, de forma que nossa contribuição vem acrescentar um novo enfoque aos estudos sobre fatores preditivos para sucesso na indução do parto. Claramente, novas 
pesquisas devem ser realizadas comparando o escore de Bishop modificado com a mensuração ecográfica do colo.

Vale salientar que encontramos área sob a curva de $71 \%$ para o escore de Bishop isolado, e de $86 \%$ quando se acrescentou a paridade. O valor desta área encontrado para o escore de Bishop assemelha-se ao descrito por Rane et al. ${ }^{13}$ para o comprimento do colo avaliado pela ultra-sonografia $(72 \%)$, sendo o valor bem superior quando se adiciona a paridade. Entretanto, estudos desenhados especificamente para comparar os dois métodos devem ser realizados, visando a elucidar esta questão que persiste não resolvida, dadas as evidências atuais.

A importância de testes preditivos para o sucesso da indução com misoprostol deve ser enfatizada. Embora o misoprostol tenha características favoráveis tanto para o preparo cervical como para a indução do parto ${ }^{31,32}$, a droga associa-se a taxas de sucesso mais elevadas nos casos com características cervicais mais favoráveis e em pacientes que já tiveram partos vaginais anteriores. Acreditamos que isto ocorre porque, em alguns casos, a indução de contrações uterinas pelo misoprostol pode se iniciar ainda com um colo imaturo, o que predisporia a freqüência mais elevada de taquissistolia e, provavelmente, de cesarianas indicadas tanto pela hiperatividade uterina como pela falta de progressão da dilatação.

Nestas situações, portanto, em que o escore de Bishop é menor que quatro, especialmente em nulíparas, seria interessante o uso de agentes préindução, para preparo cervical. O próprio misoprostol poderia ser utilizado, talvez em doses menores e intervalos maiores, permitindo o apagamento e a dilatação cervical sem, contudo, desencadear contrações uterinas clinicamente aparentes. A proposta de alguns estudos recentes diz respeito ao uso de $25 \mu \mathrm{g}$ de misoprostol, diariamente (por dois a sete dias), em regime ambulatorial ${ }^{32,33}$. Entretanto, a segurança e efetividade destes esquemas precisam ser testadas em grandes ensaios clínicos randomizados.

Até que estes estudos clínicos estejam disponiveis, nossa opinião, à semelhança da proposta de Wing et al. ${ }^{8}$, é que os esquemas de administração de misoprostol vaginal devem ser individualizados de acordo com as características de cada paciente, notadamente do escore de Bishop e da paridade, o que pode aumentar sensivelmente as taxas de sucesso da indução, resultando em maior proporção de partos vaginais.

\section{ABSTRACT}

Purpose: to determine the main factors associated with vaginal delivery in high-risk pregnant women submitted to labor induction with vaginal misoprostol $(50 \mu \mathrm{g})$.

Methods: this is a secondary analysis of an open nonrandomized clinical trial that included 61 high-risk pregnant women admitted at the "Maternidade-Escola Assis Chateaubriand", Fortaleza (Ceará). All women had singleton pregnancies with alive fetuses, gestational age $\geq 37$ weeks and Bishop scores $\leq 7$. Misoprostol was vaginally administered at doses of $50 \mu \mathrm{g}$ every $6 \mathrm{~h}$ for a maximum of four doses. Univariate and multiple logistic regression analyses were performed to determine association between vaginal delivery (dependent variable) and independent variables (predictive), and receiver operating characteristic (ROC) curves were constructed for parity and Bishop scores. Results: parity (one or more previous deliveries), Bishop scores $\geq 4$ and interval induction to delivery $<6 h$ were significantly associated with vaginal delivery, while tachysystole reduced the probability of vaginal delivery. A multivariate stepwise logistic regression was then performed to evaluate each of these as independent predictors. Parity $(O R=5.41,95 \% C I=4.18-6.64)$ and Bishop score $\geq 4(O R=$ $3.30,95 \% C I=2.15-4.45$ ) were significant independent predictors for vaginal delivery. In the ROC curve for parity and Bishop score, sensitivity of $63.2 \%$ and positive predictive value of $100 \%$ were found. The area under the ROC curve was $86.8 \%$, significantly higher than $50 \%(p=0.023)$.

Conclusions: the most important predictive factors for vaginal delivery after induction with misoprostol were parity and Bishop score. These characteristics should be considered when choosing schemes and doses of misoprostol for cervical ripening and labor induction.

KEYWORDS: Labor induction. Prostaglandins. Labor.

Conflito de interesses: não mencionados

\section{Referências}

1. Margulies M, Catuzzi P, Voto LS, Imaz FU. Inducción deI trabajo de parto con un analogo de la $\operatorname{PgE}_{1}$. Prensa Med Argent 1991; 78:9-13.

2. Alfirevic $Z$. Oral misoprostol for induction of labour. Cochrane Database Syst Rev 2003; (2):CD001338.

3. Hofmeyr GJ, Gulmezoglu AM. Vaginal misoprostol for cervical ripening and induction of labour. Cochrane Database Syst Rev 2003; (1):CD000941.

4. Tang OS, Schweer H, Seyberth HW, Lee SW, Ho PC. Pharmacokinetics of different routes of administration of misoprostol. Hum Reprod 2002; $17: 332-6$. 
5. Zieman M, Fong SK, Benowitz NL, Banskter D Darney PD. Absorption kinetics of misoprostol with oral or vaginal administration. Obstet Gynecol 1997; 90:88-92.

6. Sanchez-Ramos L, Kaunitz AM, Delke I. Labor induction with 25 microg versus 50 microg intravaginal misoprostol: a systematic review. Obstet Gynecol 2002; 99:145-51.

7. Wing DA. Labor induction with misoprostol. Am J Obstet Gynecol 1999; 181:339-45.

8. Wing DA, Tran S, Paul RH. Factors affecting the likelihood of successful induction after intravaginal misoprostol application for cervical ripening and labor induction. Am J Obstet Gynecol 2002; 186:1237-43.

9. Ware V, Raynor BD. Transvaginal ultrasonographic cervical measurement as a predictor of successful labor induction. Am J Obstet Gynecol 2000; 182:1030-2.

10.Cano A, Carrillo M, Baquero R, Lozano F, Sarmiento A, Pinzón W. Cervicometría transvaginal como factor predictor de éxito en indución del trabajo de parto con misoprostol. Rev Colomb Obstet Ginecol 2000; 51:214-7.

11.Watson WJ, Stevens D, Welter S, Day D. Factors predicting successful labor induction. Obstet Gynecol 1996; 88:990-2.

12.Gonen R, Degani S, Ron A. Prediction of successful induction of labor: comparison of transvaginal ultrasonography and the Bishop score. Eur J Ultrasound 1998; 7:183-7.

13. Rane SM, Guirgis RR, Higgins B, Nicolaides $\mathrm{KH}$. Pre-induction sonographic measurement of cervical length in prolonged pregnancy: the effect of parity in the prediction of the need for Cesarean section. Ultrasound Obstet Gynecol 2003; 22:45-8.

14.Sampaio ZS, Alencar Júnior CA, Feitosa FEL, Amorim MMR. Uso de misoprostol vaginal (50mcg) para indução do parto em gestações de alto-risco: ensaio clínico aberto. Rev Bras Saúde Matern Infant. No prelo.

15.Arcanjo FCN, Alencar Júnior CA, Feitosa FEL, Amorim MMR. Uso de misoprostol retal para indução do parto em gestantes com amniorrexe prematura: ensaio clínico fase II. Rev Bras Ginecol Obstet 2003; 25:491-9.

16.Lucena FEL, Sampaio ZS, Alencar Júnior CA, Amorim MMR, Passini R. Uso do misoprostol para indução do parto. Cad Saúde Pública. No prelo.

17.Brasil. Ministério da Saúde. DATASUS. Nascidos vivos 2003 [acesso 11 ago 2003]. Disponível em URL: http: //www.datasus.gov.br

18. Report of the National High Blood Pressure Education Program Working Group on High Blood Pressure in Pregnancy. Am J Obstet Gynecol 2000; 183:S1-S22.

19.Hadlock FP, Harrist RB, Sharman RS, Deter RL, Park SK. Estimation of fetal weight with the use of head, body, and femur measurements: a prospective study. Am J Obstet Gynecol 1985; 151:333-7.

20.Phelan JP, Smith CV, Broussard P, Small M. Amniotic fluid volume assessment with the fourquadrant technique at 36-42 weeks' gestation. J Reprod Med 1987; 32:540-2.

21.NHS Executive. Clinical guidelines: using clinical guidelines to improve patient care within the NHS. London: HMSO; 1996.

22.Royal College of Obstetricians and Gynaecologists. Induction of labor: evidence-based. Clinical Guideline Number 9. RCOG Clinical Effectiveness Suport Unit, Jun 2001. Disponivel em URL: http:// www.rcog.org.uk/resources/public/rcog_induction of_labour.pdf

23.Paterson-Brown S, Fisk NM, Edmonds DK, Rodeck $\mathrm{CH}$. Preinduction cervical assessment by Bishop's score and transvaginal ultrasound. Eur J Obstet Gynecol Reprod Biol 1991; 40:17-23.

24.Boozarjomehri F, Timor-Tritsch I, Chao CR, Fox HE. Transvaginal ultrasonographic evaluation of the cervix before labor: presence of cervical wedging is associated with shorter duration of induced labor. Am J Obstet Gynecol 1994; 171:1081-7.

25.Williams MC, Krammer J, O'Brien WF. The value of the cervical score in predicting successful outcome of labor induction. Obstet Gynecol 1997; 90:784-9.

26.Bishop EH. Pelvic scoring for elective induction. Obstet Gynecol 1964; 24:266-8.

27.Zilianti M, Azuaga A, Calderon F, Pages G, Mendoza G. Monitoring the effacement of the uterine cervix by transperineal sonography: a new perspective. J Ultrasound Med 1995; 14:719-24.

28.Tedesco RP, Cecatti JG, Maia Filho NL. Efetividade de duas diferentes doses de misoprostol por via vaginal para preparo cervical e indução do parto. Rev Bras Ginecol Obstet 2002; 24:641-6.

29.Moraes Filho OB. Misoprostol versus sonda Foley e ocitocina para indução do parto. Rev Bras Ginecol Obstet 2002; 24:685.

30.Aquino MMA, Cecatti JG. Misoprostol versus oxytocin for labor induction in term and post-term pregnancy: randomized controlled trial. São Paulo Med J 2003; 121:102-6.

31.Goldberg AB, Greenberg MB, Darney PD. Misoprostol and pregnancy. N Engl J Med 2001; 344:38-47.

32.Stitely ML, Browning J, Fowler M, Gendron RT, Gherman RB. Outpatient cervical ripening with intravaginal misoprostol. Obstet Gynecol 2000; 96:684-8.

33.Incerpi MH, Fassett MJ, Kjos SL, Tran SH, Wing DA. Vaginally administered misoprostol for outpatient cervical ripening in pregnancies complicated by diabetes mellitus. Am J Obstet Gynecol 2001; 185:916-9.

Recebido em: $27 / 10 / 2003$ Aceito com modificações em: 6/1/04 